\title{
Minimization of energy use in multipurpose batch plants using heat storage: an aspect of cleaner production
}

\author{
Thokozani Majozi* \\ Department of Chemical Engineering, University of Pretoria, Lynnwood Road, Pretoria 0002, South Africa \\ Department of Computer Science, University of Pannonia, Egyetem u. 10, Veszprém H-8200, Hungary
}

Keywords:

Cleaner production

Heat integration

Batch plants

Optimization

MILP

\begin{abstract}
A B S T R A C T
A mathematical technique for optimization of energy use through the exploitation of heat storage in heat integrated multipurpose batch plants is presented in this document. Storage of heat is effected through the use of a heat transfer fluid. The resultant mathematical formulation exhibits a mixed integer linear programming (MILP) structure, which yields a globally optimal solution for a predefined storage size. The concept of heat integration in batch plants with the objective of minimising energy use is indeed an aspect of cleaner production that has received very limited attention in published literature. This is largely because batch plants are perceived to be less energy-intensive compared to their continuous production counterparts which is untrue for some batch operations, like dairy and brewing processes; hence the need for dedicated techniques. Application of the proposed method to an agrochemical facility has shown savings of more than $75 \%$ in external utility steam consumption.
\end{abstract}

(c) 2009 Elsevier Ltd. All rights reserved.

\section{Introduction}

Until recently, heat integration has always been applied to continuous processes rather than to batch chemical processes. This is mainly due to the fact that, in general, heat integration techniques assume steady-state behaviour, which is a feature of continuous processes. Moreover, batch operations tend to be less energy-intensive than their continuous counterparts. However, the increasing popularity of batch plants and the continuing global emphasis on emissions reduction is starting to warrant either the adaptation of the well-established heat integration techniques to or the development of novel techniques for batch processes. The increase in popularity is due to the flexibility and adaptability of batch plants, which is crucial in the current, volatile market conditions. It is also worthy of note that, although external utility requirement is a secondary economic issue in most batch facilities, e.g. agrochemicals and pharmaceuticals, it can be significant in others, e.g. dairy and brewing [11].

Early work on heat integration of batch plants was proposed by Vaselanak et al. [18]. These authors explored heat integration of batch vessels containing hot fluid that required cooling and cold fluid that required heating. Four cases were investigated. In the first case, the fluid from one vessel was allowed to return to the same

\footnotetext{
* Tel.: +27 12420 4130; fax: +27 124205048

E-mail address: thoko.majozi@up.ac.za
}

vessel after exchanging heat with the fluid of another vessel via a common heat exchanger. In the second case, a heating or cooling medium was used to transfer heat between the hot fluid vessel and the cold fluid vessel, thereby, maintaining the heat integrated fluids within the vessels throughout the heat exchange process. The third case entailed the transfer of fluids from their original vessels to receiving vessels while being heated or cooled. The fourth case was the combination of the above cases. Implicit in their analyses was the given schedule of the operations. A heuristic procedure was proposed for the cases where the final temperatures were not limiting and an MILP formulation for the cases where the final temperatures were limiting.

Subsequent to this, other mathematical formulations for heat integration of batch processes have been proposed by Peneva et al. [14], Ivanov et al. [3], Corominas et al. [2], Papageorgiou et al. [13], Vaklieva-Bancheva et al. [17], and Adonyi et al. [1]. Peneva et al. [14] and Ivanov et al. [3] addressed the problem of designing a minimum total cost heat exchanger network for given pair wise matches of batch vessels. An implicit, predefined schedule was also assumed. Corominas et al. [2] considered the problem of designing a minimum cost heat exchanger network and a heat exchange strategy for multiproduct batch plants operating in a campaign mode. The objective was to maximize heat exchange in a prespecified campaign of product batches with hot streams requiring cooling and cold streams requiring heating. The emphasis on campaign mode implies that the proposed methodology cannot be applied in situations where equipment scheduling is crucial. 
Papageorgiou et al. [13] extended the discrete-time formulation of Kondili et al. [7] for scheduling of multipurpose batch plants by including heat integration aspects. Direct and indirect heat integration configurations were addressed. The main drawback of all discrete-time formulations is their explosive binary dimension, which requires enormous computational effort [16]. VaklievaBancheva et al. [17] improved the work of Ivanov et al. [3] by embedding the heat integration framework within an overall scheduling framework. However, the authors only addressed a special case in which the plant is assumed to operate in a zerowait overlapping mode, where each product must pass through a subset of the equipment stages, and production is organized in a series of long campaigns. Recently, Pinto et al. [15] presented a discrete-time mixed integer mathematical formulation for the design of heat integrated multipurpose plants based on a superstructure approach.

A graph theory based technique that incorporates heat integration within scheduling of multipurpose plants has also been proposed by Adonyi et al. [1] with emphasis to make span minimization. Other established attempts on heat integration of batch plants are based on the concept of pinch analysis [8], which was initially developed for continuous processes at steady-state. As such, these methods assume a pseudo-continuous behaviour in batch operations either by averaging time over a fixed time horizon of interest [9] or assuming fixed production schedules within which opportunities for heat integration are explored [4-6,12]. It is, therefore, evident that these methods cannot be applied in situations where a production schedule that maximizes heat integration whilst optimizing production demands is sought.

The methodology presented in this paper is an extension of the methodology developed by Majozi [19] which was only aimed at direct heat integration of batch plants. The main advantages of this methodology are that the start and end times of processes need not be specified a priori and requires very few binary variables due to uneven discretization of the time horizon of interest. The extension pertains to the inclusion of heat storage as a possibility for saving more energy and allowing overall flexibility of the process. The mathematical model is linear which implies that the solution corresponding to a predefined size of storage is globally optimal.

\section{Problem statement}

The problem addressed in this paper can be stated as follows. Given:

(i) production scheduling data, i.e. equipment capacities, task durations, time horizon of interest, recipe for each product as well as cost of raw materials and selling price of final products,

(ii) hot and cold duties for tasks that require heating and cooling, respectively,

(iii) cost of cooling water and steam,

(iv) operating temperatures for the heat source and the heat sink operations,

(v) allowed minimum temperature differences and

(vi) available heat storage capacity

determine the production schedule that results in minimum energy use or maximum profit. In the context of this paper, profit is defined as the difference between revenue and operating costs. The latter constitute raw material costs and external utility (cooling water and steam) costs. It is assumed that sufficient temperature driving forces exist between matched tasks for process-process heat transfer. Also, each task is allowed to operate either in an integrated or stand alone mode. The integrated mode, in the context of this paper, is twofold, since a unit is allowed to be

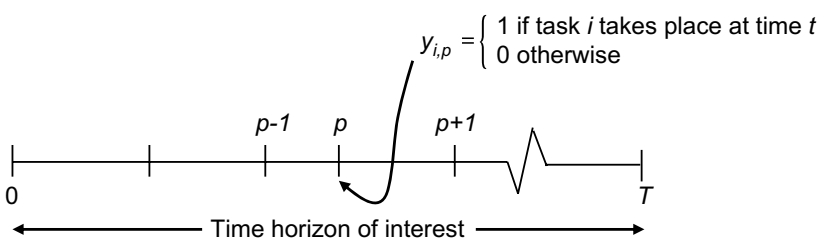

Fig. 1. Continuous time representation of the time horizon.

integrated with either heat storage or another operating unit. If heat integration cannot supply sufficient duty, external utility is supplied to complement the deficit. Whilst direct heat integration requires involved tasks to be active within a common time interval to effect direct heat transfer, they need not necessarily commence nor end at the same time. Moreover, the heat integrated tasks can either belong to the same process or to distinct processes within reasonable proximity.

\section{Mathematical model}

As aforementioned, the mathematical model proposed in this paper is an extension of the earlier work by the same author. It is based on the so called continuous time representation of the time horizon shown in Fig. 1 and entails the following sets, variables and parameters.

Sets

$U=\{u \mid u$ is a heat storage unit $\}$

$J=\{j \mid j$ is a processing unit $\}$

$J_{c}=\left\{j_{c} \mid j_{c}\right.$ is a processing unit that requires cooling $\} \subset J$

$J_{h}=\left\{j_{h} \mid j_{h}\right.$ is a processing unit that requires heating $\} \subset J$

$P=\{p \mid p$ is a time point $\}$

$S_{\text {in }, j}=\left\{s_{\text {in }, j} \mid S_{\text {in }, j}\right.$ is an input stream to a processing unit $\}$

$S_{\text {out }, j}=\left\{s_{\text {out }, j} \mid s_{\text {out }, j}\right.$ is an output stream from a processing unit $\}$

\section{Variables}

$t_{p}\left(s_{\text {out } j,}, p\right)=$ time at which the stream is produced from unit $j$ $t_{u}\left(s_{\mathrm{in}, j}, p\right)=$ time at which the stream enters the processing unit $j$ $T_{0}(u, p)=$ initial temperature in the storage vessel at time point $p$ $T_{f}(u, p)=$ final temperature in the storage vessel at time point $p$ $C W(j, p)=$ amount of external cooling required by operation $j$ at time point $p$

$S T(j, p)=$ amount of external heating required by operation $j$ at time point $p$

$Q(j, u, p)=$ amount of heat exchanged with storage at time point $p$

$y(j, u, p)=\left\{\begin{array}{l}1 \leftarrow \text { if unit } j \text { is exchanging heat with storage unit } u \\ 0 \leftarrow \text { otherwise }\end{array}\right.$

$x\left(j, j^{\prime}, p\right)=\left\{\begin{array}{l}1 \leftarrow \text { if unit } j \text { is exchanging heat with another unit } j^{\prime} \\ 0 \leftarrow \text { otherwise }\end{array}\right.$

$y\left(s_{\text {in }, j}, p\right)=\left\{\begin{array}{l}1 \leftarrow \text { if unit } j \text { is active at time point } p \\ 0 \leftarrow \text { otherwise }\end{array}\right.$

\section{Parameters}

$T(j)=$ operating temperature for processing unit $j$

$\tau(j)=$ duration of operation $j$ in standalone mode

$\tau^{\prime}\left(j, j^{\prime}\right)=$ duration of operation $j$ when directly heat integrated

$\tau^{\prime \prime}(j, u)=$ duration of operation $j$ when integrated with storage

$\Delta T^{\mathrm{min}}=$ minimum temperature difference

$Q(j)=$ amount of heat required by or removed from the operating unit $j$

$M(u)=$ capacity of heat storage $u$ 


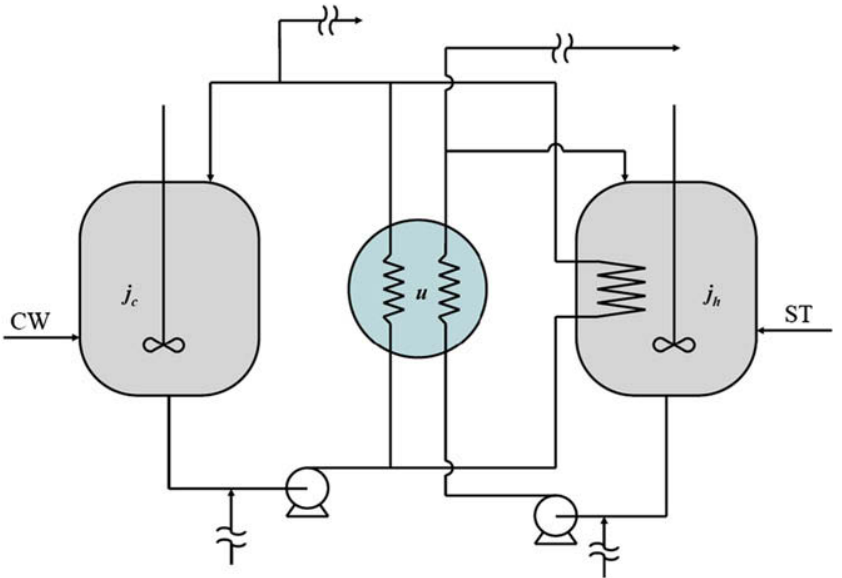

Fig. 2. Superstructure for the mathematical model.

\section{Constraints}

The mathematical model is based on the superstructure shown in Fig. 2. The heat transfer fluid in heat storage remains in the storage vessel during heat transfer with only the process fluid pumped around. The superstructure also shows that each unit is capable of receiving external heating or cooling in addition to direct and indirect heat integration.

In addition to scheduling constraints that have been presented in detail in another publication [10], the following constraints are necessary to cater for heat storage. Constraints (1) and (2) ensure that direct heat integration involves exactly one pair of units so as to simplify process operability. In essence, these constraints state that if two units are heat integrated at any given point in time, then these units must also be active at that point in time. However, if a unit is active at a given time point it is not necessary that it be heat integrated with another unit.

$\sum_{j^{\prime} \in J_{c}} x\left(j, j^{\prime}, p\right) \leq y\left(s_{\text {in }, j}, p\right), \quad \forall p \in P, j \in J_{h}, s_{\text {in }, j} \in S_{\text {in }, j}$ $\sum_{j \in J_{h}} x\left(j, j^{\prime}, p\right) \leq y\left(s_{\mathrm{in}, j^{\prime}}, p\right), \quad \forall p \in P, j \in J_{c}, s_{\mathrm{in}, j^{\prime}} \in S_{\mathrm{in}, j}$

Constraints (3)-(5) quantify the amount of heat transferred and received from storage unit, respectively. They ensure that if there is no heat integration between a processing unit and storage, then the amount of heat related to storage is not disturbed.

$$
\begin{gathered}
Q(j, u, p 0)=M(u) c_{p}\left(T_{f}(u, p 1)-T_{\text {Start }}\right) y(j, u, p 0), \quad \forall j \in J_{c} \subset J, u \in U \\
\begin{aligned}
& Q(j, u, p-1)= M(u) c_{p}\left(T_{f}(u, p)-T_{0}(u, p-1)\right) y(j, u, p-1), \\
& \forall j \in J_{c} \subset J, p \in P, p>p 0, u \in U
\end{aligned} \\
\begin{aligned}
Q\left(j^{\prime}, u, p-1\right)= & M(u) c_{p}\left(T_{0}(u, p-1)-T_{f}(u, p)\right) y\left(j^{\prime}, u, p-1\right) \\
& \forall j \in J_{h} \subset J, p \in P, p>p 0, u \in U
\end{aligned}
\end{gathered}
$$

Constraint (6) ensures that only one unit is heat integrated with storage at any given point in time. Constraints (7) and (8) ensure that the temperature of the storage unit is not changed if there is no heat integration with any unit. These constraints carry the same meaning as constraints (3)-(5). Nonetheless, they are necessary since they pertain to temperature whilst the latter pertain to the amount of heat. Overall, constraints (3)-(8) govern the relationship between heat and temperature of storage.

$$
\begin{aligned}
& \sum_{j \in J_{c}} y(j, u, p)+\sum_{j^{\prime} \in J_{h}} y\left(j^{\prime}, u, p\right) \leq 1, \quad \forall p \in P, u \in U \\
& T_{0}(u, p-1) \leq T_{f}(u, p)+\max _{j}\{T(j)\} \\
& \quad\left(\sum_{j \in J_{c}} y(j, u, p-1)+\sum_{j^{\prime} \in J_{h}} y\left(j^{\prime}, u, p-1\right)\right), \quad \forall p \in P, p>p 0, u \in U
\end{aligned}
$$

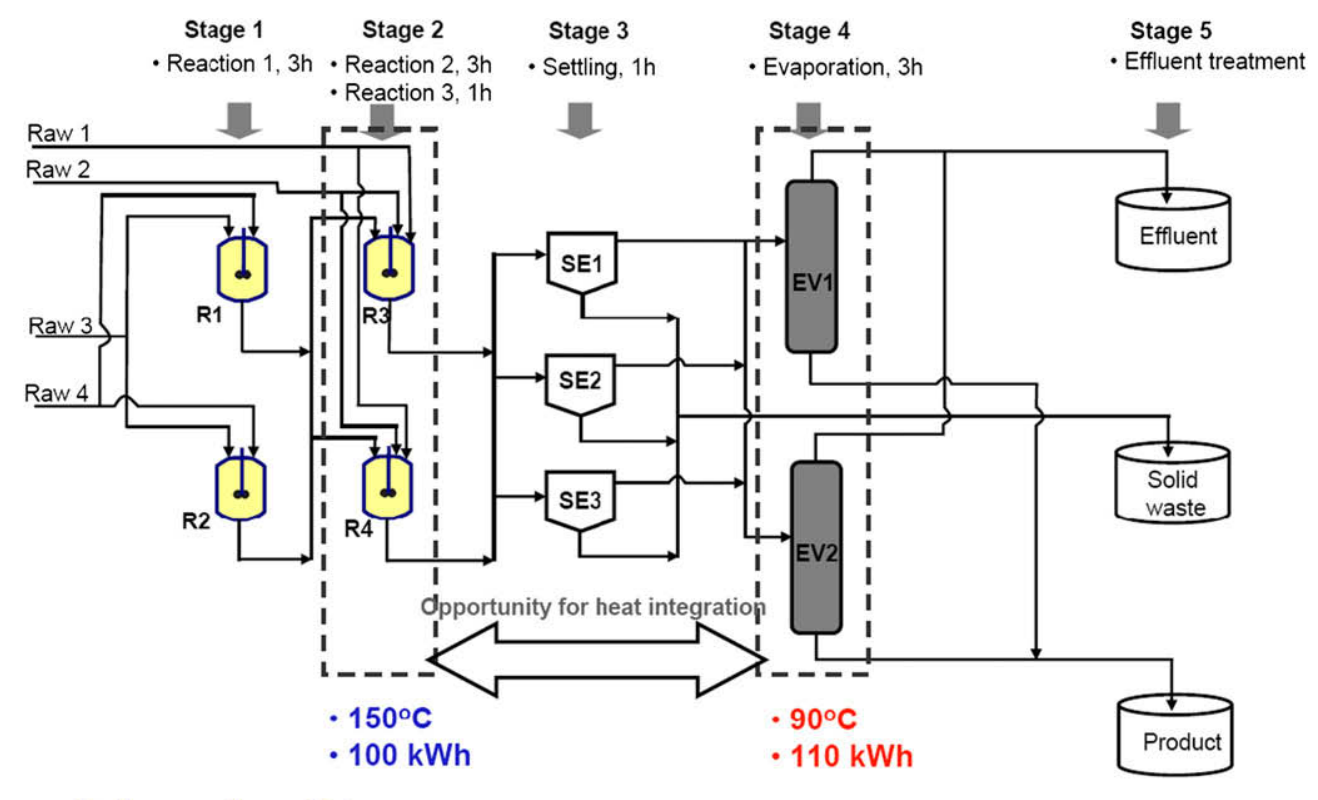

- Unit capacity $=10$ tons

Fig. 3. Process flowsheet for the case study. 
Table 1

Data for the case study.

Specific heat capacity of water $c_{p}\left(\mathrm{~kJ} / \mathrm{kg}{ }^{\circ} \mathrm{C}\right)$

Latent heat of vaporization $\lambda_{v}(\mathrm{~kJ} / \mathrm{kg})$

Product cost (cu/ton)

Steam cost (cu/kWh)

Cooling water cost $(\mathrm{cu} / \mathrm{kWh})$

$T_{0}(u, p-1) \geq T_{f}(u, p)-\max _{j}\{T(j)\}$

$$
\left(\sum_{j \in J_{c}} y(j, u, p-1)+\sum_{j^{\prime} \in J_{h}} y\left(j^{\prime}, u, p-1\right)\right), \forall p \in P, p>p 0, u \in U
$$

Constraint (9) ensures that the initial temperature in heat storage at any given point in time is the same as the final temperature at the last time point. This condition is always true, regardless of the heat integration status in the previous time point.

$T_{0}(u, p)=T_{f}(u, p-1), \quad \forall p \in P, u \in U$

Constraints (10) and (11) ensure that if there is heat integration between any unit and heat storage, then the stipulated minimum driving force should be obeyed. Constraint (10) applies if heat storage is integrated with the heat source, whilst constraint (11) applies if heat storage is integrated with the heat sink.

$$
\begin{gathered}
T(j)-T_{f}(u, p) \geq \Delta T^{\min }-\max _{j}\{T(j)\}(1-y(j, u, p-1)), \\
\forall p \in P, p>p 0, j \in J_{c} \subset J, u \in U \\
T_{f}(u, p)-T(j) \geq \Delta T^{\min }-\max _{j}\{T(j)\}(1-y(j, u, p-1)), \\
\forall p \in P, p>p 0, j \in J_{h} \subset J, u \in U
\end{gathered}
$$

Constraint (12) states that cooling in any heat source will be accomplished either by direct heat integration, external cooling or heat integration with storage. Constraint (13) is similar to constraint (12) but applies to a heat sink.

$$
\begin{aligned}
Q(j) y\left(s_{\mathrm{in}, j}, p\right)= & Q(j, u, p)+C W(j, p)+\sum_{j^{\prime} \in J_{h}} \min _{j, j^{\prime}}\left\{Q(j), Q\left(j^{\prime}\right)\right\} x\left(j, j^{\prime}, p\right), \\
& \forall j \in J_{c} \subset J, p \in P, u \in U \\
Q(j) y\left(s_{\mathrm{in}, j}, p\right)= & Q(j, u, p)+S T(j, p)+\sum_{j \in J_{c}} \min _{j, j^{\prime}}\left\{Q(j), Q\left(j^{\prime}\right)\right\} x\left(j, j^{\prime}, p\right), \\
& \forall j \in J_{h} \subset J, p \in P, u \in U
\end{aligned}
$$

Table 2

Summary of results from the case study.

\begin{tabular}{llll}
\hline & $\begin{array}{l}\text { No heat } \\
\text { integration }\end{array}$ & $\begin{array}{l}\text { Direct heat } \\
\text { integration }\end{array}$ & $\begin{array}{l}\text { Heat } \\
\text { storage }\end{array}$ \\
\hline $\begin{array}{l}\text { Performance index } \\
\quad \text { (cost units) }\end{array}$ & 130,200 & 137,000 & 138,600 \\
$\begin{array}{l}\text { External cold duty }(\mathrm{kW}) \\
\text { External hot duty (kW) }\end{array}$ & 400 & 300 & 100 \\
CPU time (s) & 330 & 30 & 30 \\
Constraints & $\mathbf{1 8 2 . 7 3}$ & $\mathbf{2 6 . 8}$ & $\mathbf{2 8 0 5 . 2}$ \\
$\begin{array}{l}\text { Continuous variables } \\
\text { Binary variables }\end{array}$ & & & 4144 \\
\hline
\end{tabular}

a Performance index $=$ revenue - utility costs.

b Results obtained from GAMS/CPLEXPAR 7.0 using Intel Core 2 Duo $1.2 \mathrm{GHz}$ processor.
Constraints (14) and (15) state that if a unit is directly heat integrated with another unit, then it cannot be simultaneously integrated with heat storage. This is also a condition imposed solely to simplify operability of the overall process.

$\sum_{j^{\prime} \in J_{h}} x\left(j, j^{\prime}, p\right)+y(j, u, p) \leq 1, \quad \forall j \in J_{c} \subset J, p \in P, u \in U$

$\sum_{j \in J_{c}} x\left(j, j^{\prime}, p\right)+y\left(j^{\prime}, u, p\right) \leq 1, \quad \forall j^{\prime} \in J_{h} \subset J, p \in P, u \in U$

Constraint (16) is a feasibility constraint which ensures that if a unit is not integrated with storage, then the associated duty should not exist.

$\delta y(j, u, p) \leq Q(i, u, p) \leq \max _{j \in J}\left(Q(j), Q\left(j^{\prime}\right)\right) y(j, u, p)$

Constraint (17) shows how the variation in duration due to the heat integration mode is accounted for in the mathematical model. It is very likely that the duration times will be affected by the mode of operation and this should not be ignored in the formulation.

$$
\begin{aligned}
t_{p}\left(s_{\text {out }, j}, p\right)= & t_{u}\left(s_{\text {in }, j}, p-1\right)+\tau(j)\left(1-y\left(j, j^{\prime}, p\right)-y(j, u, p)\right) \\
& +\tau^{\prime \prime}(j, u) y(j, u, p)+\tau^{\prime}\left(j, j^{\prime}\right) y\left(j, j^{\prime}, p\right) \\
& \forall j \in J, p \in P, u \in U, s_{\text {in }, j}, s_{\text {out }, j} \in S
\end{aligned}
$$

The foregoing constraints constitute the full heat storage model. With the exception of constraints (3)-(5), all the constraints are linear. Constraints (3)-(5) entail nonconvex bilinear terms which render the overall model a nonconvex MINLP. However, the type of bilinearity exhibited by these constraints can be readily removed without compromising the accuracy of the model using the so called Glover transformation. This is demonstrated underneath using constraint (3).

$$
\begin{gathered}
Q(j, u, p)=M(u) c_{p}\left(\Gamma_{f}(j, u, p)-\Gamma_{0}(j, u, p)\right), \\
\forall j \in J_{c} \subset J, p \in P, p>p 0, u \in U \\
T_{f}(u, p)-T_{f}^{U}(u)(1-y(j, u, p-1)) \leq \Gamma_{f}(j, u, p) \\
\leq T_{f}(u, p)+T_{f}^{L}(u)(1-y(j, u, p-1)), \\
\forall j \in J_{c} \subset J, p \in P, p>p 0, u \in U
\end{gathered}
$$

$T_{f}^{L}(u) y(j, u, p-1) \leq \Gamma_{f}(j, u, p) \leq T_{f}^{U} y(j, u, p-1)$,

$$
\forall j \in J_{c} \subset J, p \in P, p>p 0, u \in U
$$

$$
\begin{aligned}
T_{0}(u, p-1)- & T_{0}^{U}(u)(1-y(j, u, p-1)) \leq \Gamma_{0}(j, u, p) \\
& \leq T_{0}(u, p-1)+T_{0}^{L}(u)(1-y(j, u, p-1)), \\
& \forall j \in J_{c} \subset J, p \in P, p>p 0, u \in U
\end{aligned}
$$

$T_{0}^{L}(u) y(j, u, p-1) \leq \Gamma_{0}(j, u, p) \leq T_{0}^{U} y(j, u, p-1)$,

$$
\forall j \in J_{c} \subset J, p \in P, p>p 0, u \in U
$$

Constraints (19)-(21) constitute the linearized version of constraint (3). The advantage of this linearization technique is that it is exact, which implies that global optimality is assured. The disadvantage, however, is that it requires the introduction of new variables and additional constraints. Consequently, the size of the model is increased. A similar type of linearization is also necessary for constraint (4) in order to have an overall MILP model which can be solved exactly to yield a globally optimal solution. 


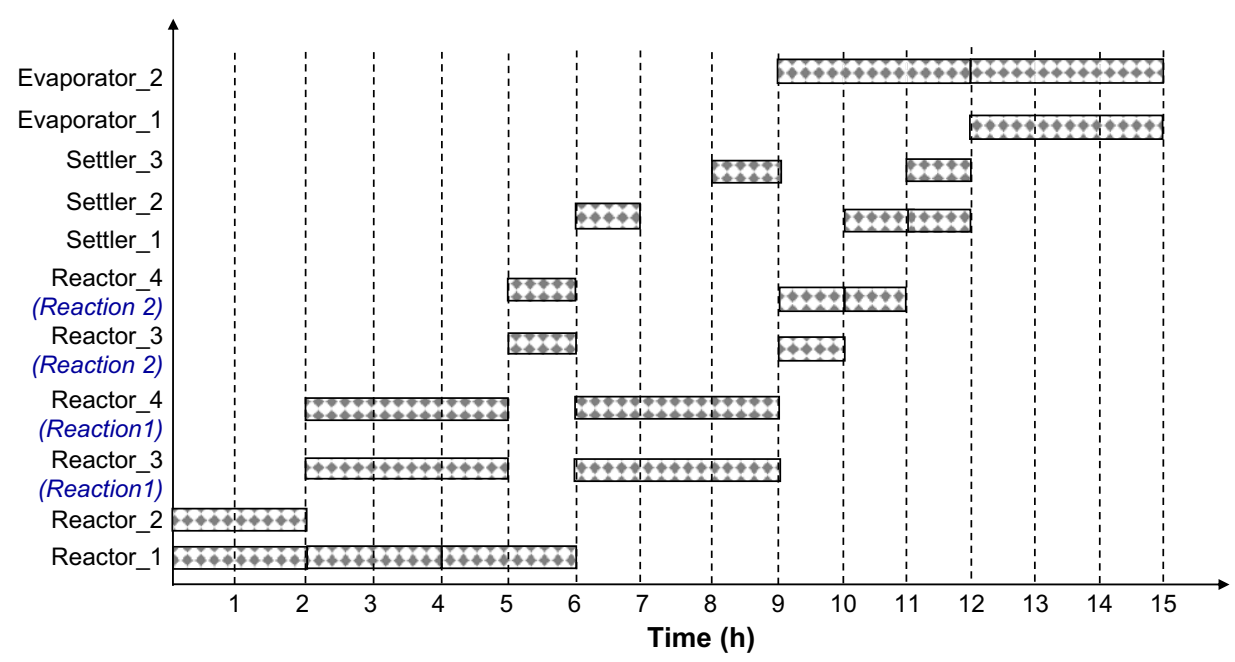

Fig. 4. Gantt chart for the case study without heat integration.

\section{Case study}

Fig. 3 is the representation of the case study that was used to demonstrate the performance of the proposed model it is taken from the literature [19]. To facilitate understanding, this case study is described in some detail in this paper. The plant, which constitutes $30 \%$ of production and consumes $55 \%$ of utility steam in the multinational agrochemical facility, involves the manufacture of an herbicide. The saturated steam is produced from a coal fired boiler at 10 bar absolute pressure and three $\mathrm{t} / \mathrm{h}$, although it is used at $4 \mathrm{bar}$ in the chosen process. The process entails three consecutive chemical reactions which take place in four reactors. The first reaction, which uses water as a solvent, takes place in reactors $\mathrm{R} 1$ and $\mathrm{R} 2$. The two raw materials shown in the flowsheet are added manually, almost simultaneously, through the chutes. The intermediate from this reaction is then transferred to reactors R3 and R4, wherein two consecutive reactions take place. The first of these two reactions is highly exothermic and requires almost nine tons of cooling water (equivalent to $100 \mathrm{kWh}$ ). For operational purposes, these two consecutive reactions take place in a single reactor. However, some of the intermediate from the first of these two reactions can be stored in an intermediate buffer tank prior to the final reaction to improve throughput. Both the second and the third reactions form sodium chloride $(\mathrm{NaCl})$ as a byproduct. Consequently, the intermediate from the third reaction is transferred to settlers SE1, SE2 and SE3, to separate $\mathrm{NaCl}$ from the supernatant aqueous solution containing the active ingredient. The salt-free solution is then transferred to evaporators where steam (equivalent to $110 \mathrm{kWh}$ ) is used to remove excess water from the product. This excess water is finally dispensed with as effluent, whilst the final product is collected in storage tanks before final formulation, packaging and transportation to customers. All unit operations in this process are at almost atmospheric pressure. The task durations are shown in the flowsheet, Fig. 3. Each of the units has a capacity of ten tons.

The temperatures for the exothermic second reaction $\left(150^{\circ} \mathrm{C}\right)$ and endothermic evaporation in stage $4\left(90^{\circ} \mathrm{C}\right)$, allow for possible heat integration. Consequently, the foregoing mathematical model was applied to this case study. The capacity of storage was fixed at two tons and the chosen initial temperature was $80^{\circ} \mathrm{C}$. It is should be mentioned at this stage that the choice of storage and initial temperature will always have an influence on optimality of energy use. Therefore, these should be optimization variables, instead of parameters. However, this will be subject of a subsequent

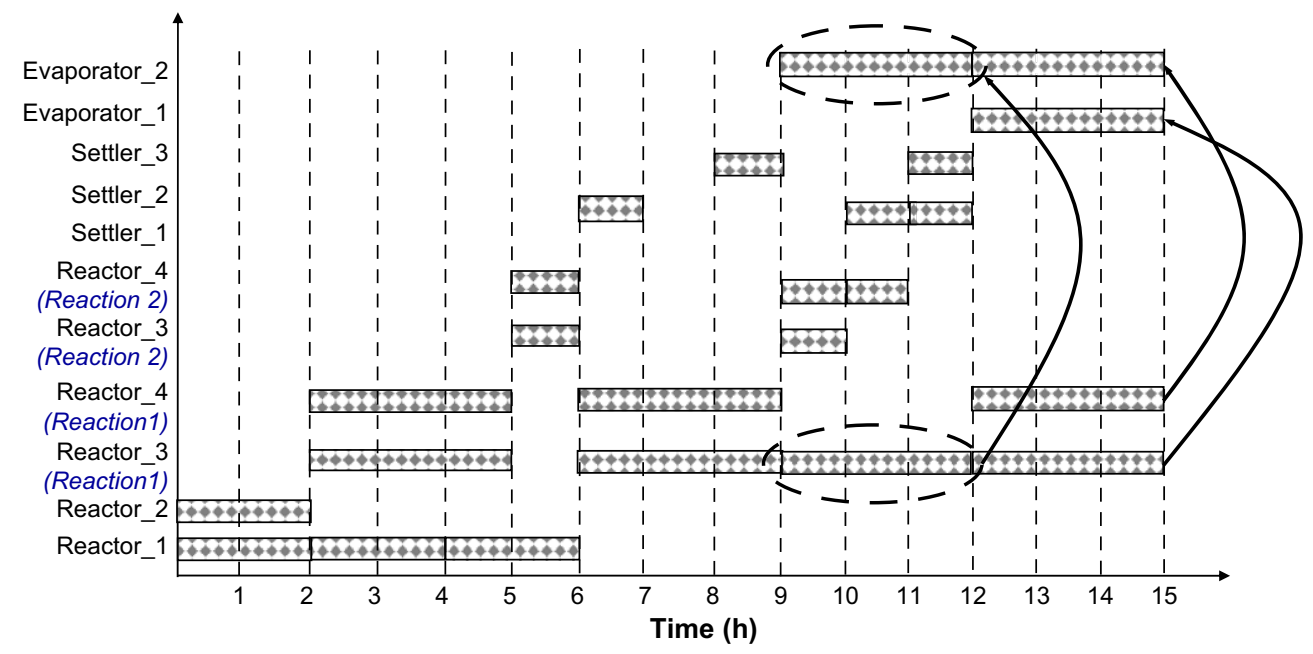

Fig. 5. Gantt chart for the case study with direct heat integration. 


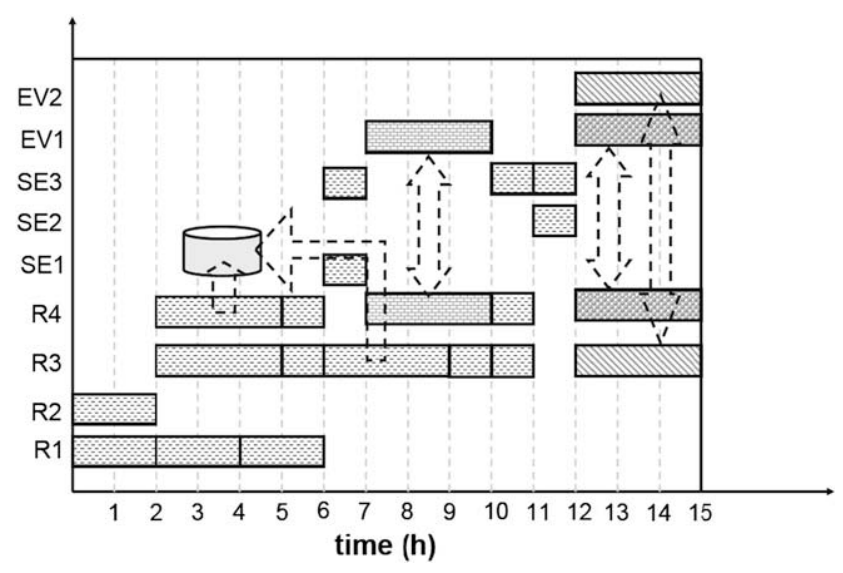

Fig. 6. Gantt chart for the case study with heat storage.

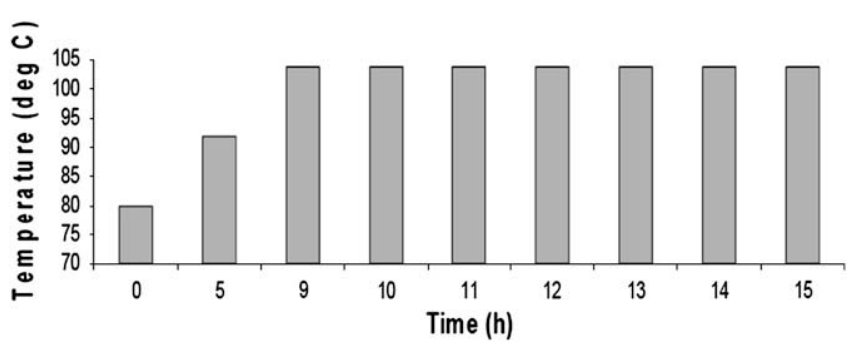

Fig. 7. Temperature profile of heat storage.

publication. The other relevant information pertaining to the case study is shown in Table 1.

\section{Results}

Whilst direct heat integration, i.e. without any use of heat store, resulted in $25 \%$ improvement in terms of external cold utility requirements, use of heat storage showed more than $75 \%$ improvement. In this particular case study, both direct and indirect heat integration resulted in $90 \%$ reduction in external hot duty requirements. These results are summarised in Table 2 . The Gantt charts corresponding to the optimal solutions for standalone operation, direct heat integration and heat integration with storage are shown in Figs. 4-6. The arrows in Figs. 5 and 6 show opportunities for direct and indirect heat integration. In Fig. 6, storage is used as heat sink by reactor R4 during time period $2-5 \mathrm{~h}$ and also by reactor R3 during time period 6-9 h. the other three process integration options involve direct heat integration. This Gantt chart is strictly a consequence of the choice of initial storage temperature and storage capacity. Fig. 7 shows the temperature profile in the storage unit over the time horizon of interest.

\section{Conclusions}

A mathematical approach for optimization of energy use in heat integrated multipurpose batch plants has been developed and tested in a case study. The results confirm that heat integration with heat storage considerations can result in energy savings of more than $75 \%$, compared to stand alone operations that rely solely on external utilities. Moreover, the option of heat storage improves the flexibility of the overall process, since the heat source and the heat sink need not take place during a common time interval.

\section{References}

[1] Adonyi R, Romero J, Puigjaner L, Friedler F. Incorporating heat integration in batch process scheduling. Appl Therm Eng 2003;23(14):1743-62.

[2] Corominas J, Espuna A, Puigjaner L. A new look at energy integration in multiproduct batch processes. Comput Chem Eng 1993;17S:S15-20.

[3] Ivanov B, Peneva K, Bancheva N. Heat integration of batch vessels at fixed time interval. I. Schemes with recycling main fluids. Hung J Ind Chem 1992;20: 225-31.

[4] Kemp IC, Deakin AW. The cascade analysis for energy and process integration of batch processes. Part 1. Calculation of energy targets. Chem Eng Res Des 1989;67:495-509.

[5] Kemp IC, MacDonald EK. Energy and process integration in continuous and batch processes. Inst Chem Eng Symp Ser 1987;105:185-200.

[6] Kemp IC, MacDonald EK. Application of pinch technology to separation, reaction and batch processes. Inst Chem Eng Symp Ser 1988;109:239-57.

[7] Kondili E, Pantelides CC, Sargent RWH. A general algorithm for short-term scheduling of batch operations. Part I-MILP formulation. Comput Chem Eng 1993; 17:211-27.

[8] Linnhoff B, Mason DR, Wardle I. Understanding heat exchanger networks. Comput Chem Eng 1979;3:295-302.

[9] Linnhoff B, Ashton GJ, Obeng EDA. Process integration of batch processes. Inst Chem Eng Symp Ser 1988;109:221-37.

[10] Majozi T, Zhu XX. A novel continuous time MILP formulation for multipurpose batch plants. 1. Short-term scheduling. Ind Eng Chem Res 2001;40(25): 5935-49.

[11] Mignon D, Hermia J. Using BATCHES for modeling and optimizing the brewhouses of an industrial brewery. Comput Chem Eng 1993;17S:S51-6.

[12] Obeng EDA, Ashton GJ. On pinch technology based procedures for the design of batch processes. Chem Eng Res Des 1988;6:255-9.

[13] Papageorgiou LG, Shah N, Pantelides CC. Optimal scheduling of heat-integrated multipurpose plants. Ind Eng Chem Res 1994;33:3168-86.

[14] Peneva K, Ivanov B, Bancheva N. Heat integration of batch vessels at fixed time interval. II. Schemes with intermediate heating and cooling agents. Hung J Ind Chem 1992;20:233-9.

[15] Pinto T, Novais AQ Barbosa-Póvoa APFD. Optimal design of heat-integrated multipurpose batch facilities with economic savings in utilities: a mixed integer mathematical formulation. Ann Oper Res 2003;120(1-4):201-30.

[16] Schilling G, Pantelides CC. A simple continuous-time process scheduling formulation and a novel solution algorithm. Comput Chem Eng 1996;20:S1221-6.

[17] Vaklieva-Bancheva N, Ivanov BB, Shah N, Pantelides CC. Heat exchanger network design for multipurpose batch plants. Comput Chem Eng 1996;20(8):989-1001.

[18] Vaselenak JA, Grossmann IE, Westerberg AW. Heat integration in batch processing. Ind Eng Chem Process Des Dev 1986;25:357-66.

[19] Majozi T. Heat integration of multipurpose batch plants using a continuoustime framework. Appl Therm Eng 2006;26:1369-77.

Thokozani Majozi is a full professor in the Department of Chemical Engineering at the University of Pretoria, South Africa, and Department of Computer Science at University of Pannonia, Hungary. His main research interest is batch process integration. His major contributions to research to date are the development of a continuous-time framework for the synthesis of batch plants and a novel technique for near zeroeffluent batch chemical facilities. Both these contributions have been adopted by industry. He started his professional career as a junior process engineer at Unilever in 1994. Before joining the University of Pretoria in 2004, and the University of Pannonia in 2006, he was the Technology Leader for Optimisation and Integration at Saso Technology, South Africa. Majozi completed his BSc degree (Eng) in 1994 and his MSc degree (Chemical Engineering) in 1998 at the University of Natal. In 2002 he completed his PhD (Chemical Engineering) at the University of Manchester Institute of Science and Technology in the United Kingdom (UK) as a recipient of the Commonwealth Scholarship. Majozi is author or co-author of more than 70 publications in scientific journals and refereed conference proceedings and is a regular speaker and session chairman at national and international conferences. 